\title{
El léxico aragonés en relación con el catalán de Valencia durante los siglos XVIII, XIX y XX
}

The Aragonese Vocabulary in Relation with the Catalan of Valencia During the 18th, 19th, and zoth Centuries

JoAquim Martí Mestre

Departament de Filologia catalana

Universitat de València

Avenida de Blasco Ibáñez, 32. València, 46010

joaquin.marti@uv.es

Orcid ID 0000-0001-5124-5225

Resumen: En este trabajo se analizan comparativamente una serie de palabras procedentes de textos costumbristas aragoneses y valencianos de los siglos XVIII, XIX y XX. El objetivo es estudiar el contacto histórico entre el léxico aragonés y el catalán, situados en un contexto peninsular y románico, relación en la cual participan otras hablas orientales peninsulares, y demostrar que la influencia histórica que ha ejercido el aragonés sobre el valenciano es mayor que la que a veces muestran los diccionarios etimológicos e históricos. Asimismo, se aportan nuevos datos y correcciones a la bibliografía existente, así como voces valencianas no registradas (coscarrana, a tassament), primeras documentaciones (acabacases, bolxaca, cara-sol, cútio, estrela, a la gatamau, monyica) y vocablos de uso en Aragón no registrados en el DECH (acabacasas, bolchaco, botinchado, a gatomiau, tasamente), o registrados pero no en este territorio (botinchado, carasol). Para ello se utiliza un método basado en la geolingüística y en la documentación.

Palabras clave: Dialectología comparada. Aragonés. Catalán. Léxico. Siglos XVIII, XIX, XX.
RECIBIDO: 15 DE FEBRERO DE 2017 ACEPTADO: 5 DE MAYO DE 2017
Abstract: The purpose of this paper is to study the historical contact between Aragonese and Catalan lexicon, in the context of the Romance languages, taking into account the historical influence exerted by the Aragonese on the Valencian language, which is also influenced by other Eastern peninsular dialects. We have used a method based on Geolinguistics and documentation, in order to analyze some words from popular authors from Valencia and Aragon of the XVIII ${ }^{\text {th }}, X I X^{\text {th }}$ and $X X^{\text {th }}$ centuries. This way, our study provides new data and new interpretations on the study of the linguistic contact between the peninsular languages. Also the study provides Valencian words not included in the dictionaries (coscarrana, a tassament), first documentations (acabacases, bolxaca, cara-sol, cútio, estrela, a la gatamau, monyica) and words used in Aragón not included in the DECH (acabacasas, bolchaco, botinchado, a gatomiau, tasamente), or included in the DECH but not in Aragón (botinchado, carasol).

Keywords: Comparative Dialectology. Aragonese. Catalan. Lexicon. 18th, 19th, 20th Centuries. 
El CONTACTO HISTÓRICO ENTRE EL ARAGONÉS Y EL CATALÁN DE VALENCIA

E 1 País Valenciano es uno de los territorios donde mejor se puede ejemplificar el contacto histórico entre el aragonés y el catalán. ${ }^{1} Y$ ello de diversas formas. Por una parte, el sector occidental del territorio valenciano, repoblado mayoritariamente por aragoneses, es actualmente de lengua castellana pero con un fuerte substrato aragonés, donde se aprecia también la interferencia del catalán de Valencia. En la zona de lengua catalana, donde hubo un predominio de pobladores catalanes, participaron también en la repoblación proporciones importantes de aragoneses, y este flujo poblacional aragonés se ha venido manteniendo durante los siglos siguientes, prácticamente hasta nuestros días. A esto hay que sumar la influencia de adstrato entre territorios vecinos y el importante intercambio económico y comercial que históricamente ha existido entre las tierras aragonesas y valencianas. Por todo ello, desde la creación del antiguo reino de Valencia, el aragonés ha actuado directamente sobre el catalán de Valencia, y también indirectamente en la transmisión de castellanismos y en la familiarización de los valencianos con el castellano.

Con todo, no siempre resulta fácil valorar la influencia del aragonés sobre el valenciano. No olvidemos la contigüidad geográfica entre el aragonés y el catalán occidental, que hace difícil discernir cuándo un fenómeno lingüístico común a ambas variedades lingüísticas sería un préstamo o un caso de continuidad de área lingüística. En los dialectos constitutivos esta continuidad de área puede dar lugar, sobre todo en el léxico, a formas comunes en las variedades implicadas. En el caso del valenciano, dialecto consecutivo integrado dentro del catalán occidental, Veny proponía un método basado en la Geografía lingüística y en la documentación para identificar los posibles aragonesismos. Según este método, cuando un elemento lingüístico aparece solo en valenciano, o también en tortosino, y no se registra en los dialectos matrices septentrionales, y sí, en cambio, en los aragoneses, se puede pensar en una probable influencia de estos. Este criterio se debería complementar con la cro-

1. Se entiende por aragonés tanto el lenguaje de los textos aragoneses antiguos, denominado por algunos estudiosos "aragonés común" (Enguita 57-58), como las hablas altoaragonesas, y también recibe con frecuencia este nombre, aunque para algunos investigadores de forma impropia, la variedad regional del español hablada en Aragón, que conserva algunos elementos, sobre todo léxicos, propiamente aragoneses. Ver Alvar (7-21, 132-44); Lapesa (492-95), que se refiere a la última variedad con el nombre de castellanoaragonés; Frago (115-30) y Nagore (2002, 14-15).

2. Como veremos más adelante, algunos de los castellanismos transmitidos al valenciano por vía aragonesa han acabado siendo arcaísmos conservados en aragonés. 
nología y con la consideración formal de los elementos lingüísticos, teniendo en cuenta las características fonéticas y morfológicas de cada variedad. Así, en relación con el léxico, en valenciano nos toparíamos, por una parte, con formas compartidas con el aragonés que forman parte de un fondo léxico catalanoaragonés común, o fruto de antiguos préstamos, que se encuentran también en los dialectos constitutivos, y, por otra parte, con voces de origen aragonés peculiares del valenciano, compartidas a veces con el tortosino y con las hablas catalanas del Bajo Aragón, que participan con frecuencia de las soluciones valencianas.

Sin embargo persistirían algunas dificultades, teniendo en cuenta, como observa Casanova (2002, 1663-64), la falta de documentación de muchos fenómenos en la Edad Media y la similitud estructural y evolutiva entre el catalán y el aragonés. Por otra parte, podemos añadir que la historia de muchas palabras presenta aún lagunas que los diccionarios históricos y etimológicos no siempre aclaran, y no solo en la época medieval. En este sentido, se hace necesario mejorar el conocimiento documental de nuestras lenguas en todos los periodos de su evolución, no únicamente con relación a la época medieval, lo cual nos ayudará a poder trazar con mayor precisión la historia de las palabras, como veremos con algunos ejemplos.

Así, partiendo de textos costumbristas aragoneses y valencianos de los siglos XVIII, XIX y XX, ${ }^{3}$ se analizan una serie de palabras representativas que, como veremos, encarnan diversas posibilidades en la relación entre el valenciano y el aragonés, las cuales se hallan en los escritos de ambas procedencias y cuyo estudio aporta nuevos datos y correcciones a la bibliografía precedente, sobre la relación léxica entre el aragonés y el valenciano, y en relación con las informaciones del DECH y del DECat. Como veremos, los textos que ahora citamos, que con frecuencia representan las primeras documentaciones de las voces valencianas, $\mathrm{y}$ a veces las únicas conocidas, confirman la influencia his-

3. La literatura costumbrista trata de reproducir los detalles cotidianos de la sociedad. El lenguaje es, precisamente, uno de los medios que utiliza en la búsqueda de la verosimilitud, un lenguaje que se adecue a los personajes y a los ambientes descritos. Este modelo lingüístico permite aproximarse a la lengua hablada popular, sin olvidar, evidentemente, que se trata de una creación literaria, y que siempre hay una parte de artificio. Justes Carilla/Vázquez Obrador (31-32), en su estudio de los aragonesismos de Crispín Botana, ya señalan el interés de este tipo de literatura costumbrista para conocer el "vocabulario aragonés", y consideran "que sería conveniente realizar un estudio lingüístico exhaustivo de toda la producción baturrista, pues solo de esta forma podría establecerse el grado de difusión aproximado de que gozaban los términos aragoneses", y se podrían señalar los "términos no registrados en diccionarios, vocabularios, repertorios, o alguno que aun estándolo, tuviera distinto significado". 
tórica del aragonés sobre el valenciano, bien mediante préstamos de origen aragonés, bien de voces castellanas transmitidas al valenciano a través de esta variedad lingüística. Por otra parte, vemos que en los vocablos aquí estudiados, compartidos por el catalán y el aragonés, los diccionarios históricos y etimológicos no tienen en cuenta, o no lo hacen de manera suficiente, esta relación. Los estudiamos de forma comparativa, considerando la información de los diccionarios históricos y etimológicos, de los diccionarios normativos, de las bases de datos, de los atlas lingüísticos, así como de los repertorios y de los estudios sobre léxico catalán y aragonés, sin olvidar las otras variedades lingüísticas románicas, especialmente el español general y el occitano.

Se estudian voces aragonesas no registradas en el DECH (acabacasas, bolchaco, botinchado, a gatomiau, tasamente), voces de uso en Aragón que los diccionarios etimológicos (DCVB, DECH, DECat) registran pero no como aragonesas (botinchado, carasol), voces valencianas no recogidas en los diccionarios históricos y etimológicos catalanes (coscarrana, a tassament), así como primeras documentaciones respecto a estos diccionarios (acabacases, bolxaca, cara-sol, cútio, estrela, a la gatamau, monyica).

\section{VOCES ARAGONESAS COMPARTIDAS CON EL VALENCIANO EN LAS CUALES NO SE HABÍA TENIDO BASTANTE EN CUENTA UNA POSIBLE INFLUENCIA ARAGONESA SOBRE LA FORMA VALENCIANA}

\section{bolchaco, bolchaca / bolxaca}

hace muchos días que lo llevo rodando po los bolchacos. (Botana, serie quinta 97)

y la qui és tan pobretona / qu.en un sou junt may se veu, / puix n'i ha com a poetes, / en la boltjaca de vent, / eixa de res se previnga (Romanç nou, molt graciós... 3); encomensa a rechirar-me les bolchaques y els bolchacons de la casaca y del chopetí. (El Mole 1840-1841, 351)

Según Coromines (DECH I 701, DECat II 358), la forma bolchaca es propia del aragonés y del murciano. Este autor la documenta desde el Diccionario de

\footnotetext{
4. Transcribimos los textos aragoneses manteniendo todas las características gráficas del original, y los valencianos, basándonos en las normas de edición de la prestigiosa colección Els Nostres Clàssics de la editorial Barcino.

5. En este caso, como vemos, junto con el derivado bolchacó.
} 
Autoridades (1726). En efecto, en este diccionario, bolchaca o bolchaco 'bolsillo grande como los de las casacas y chupas', "es voz usada en Aragón, pero solamente del vulgo" (NTLLE), ${ }^{6}$ y pocos años antes bolchaca y bolchaco se encuentran en el Borrador de Siesso de Bolea, como voces aragonesas, equivalentes al español bolsillo, faltriquera o bolsa (Aliaga 2008, 100). También se conoce en Aragón la variante borchaca (Altaba 21), que se encuentra asimismo en valenciano. En sentido figurado, bolchacas 'hombre perezoso, descuidado, de poca energía' (Moneva 101), parecido al valenciano bolxaques 'home dèbil de caràcter; persona indolent' (Martí 2006, 91). Coromines (DECat II 358) cree que la $l$ de la forma valenciana pudo estar favorecida por el castellano bolsillo, pero, a la vista del aragonés bolchaca, bolchaco, creemos que más bien hay que considerar la relación con esta forma. En valenciano se documenta, como vemos en la primera de las citas de más arriba, desde el siglo XVIII.?

\section{botinchado, botinflado / botinflat, botunflat}

Conozco que me estoy poniendo como botinchada, que me se altera el pulso y me duele bastante la cabeza (Botana, serie segunda 649); Dende que ví a mi morena / mi hallo triste y botinchado, / y paice que la cangrena / mestá chupando el higádo (Botana, serie cuarta 58); tomaré una miaja é retacía pa ver si me saca estos aires que tengo encerraus en la tripa. Mencuentro así como botinchada y flatolenca, y a dos por tres me dan unos escaldafríos, que me pongo a titiritiar. (Botana, serie quinta 128)

Per això no se cumpliran els teus dichos, no, Satanàs rabut, no.t riuràs d' $E l$ Mole [...]. Y miren vostedes el butumflat! No parlen, tenint més motiu, El Turia, El Mercantil, El Poble, que els ham ficat en bugada dos voltes, sinse acrimònia, y sols de bon humor, y el gos albelloner és el que es queixa y vol en son insendiari paperet tirar al Mole (El Mole 1837, 126); Diré qu.és un sac de guano, / gros, bascós y botinflat. (Cester, en Llombart 66)

Los diccionarios académicos solo dan cuenta de botinchado en el Diccionario bistórico (1936), que lo recoge con los sentidos de 'henchido, inflado como un

6. El masculino bolchaco no figura en el DECH ni en el DECat, pero se encuentra en repertorios léxicos aragoneses. La definición 'bolsillo grande' desaparece en la segunda edición de este diccionario (1770), donde se define simplemente como 'bolsillo o faltriquera', y la referencia al tamaño del objeto ya no aparecerá en las ediciones sucesivas del DRAE.

7. Los diccionarios históricos catalanes (DCVB, DECat) no recogen el valenciano bolxaca hasta principios del siglo XX en Martí Gadea. 
boto' y figuradamente 'finchado o amoscado'. El primero lo documenta en los Puntos escripturales de las divinas letras y santos padres (Barcelona 1618) del catalán Tomás Ramón y Samenter, ${ }^{8}$ y en el aragonés Benito Coll, 9 y la segunda acepción, en la Colección de voces aragonesas de Jordana (1916). ${ }^{10}$ Siesso de Bolea, en el primer cuarto del siglo XVIII, registraba como aragonés botinchado "el apitonado; el que se enfada de qualquier cosilla. Porque suele hinchar los carrillos, como el que va a henchir de ayre un boto o pellejo. En castellano, abotijonado, está hecho un botijón" y botico binchado "el que está enojado. En Castilla, botijón", juntamente con la locución bacer el botico 'enojarse' (Aliaga 2008, 102). ${ }^{11}$ En Peralta (10) encontramos por vez primera el compuesto con inflado, botinflado 'hinchado', con el mantenimiento del grupo -NFL- (INFLARE); igualmente en Borao (130), pero este lexicógrafo le añade la acepción 'hombre desproporcionadamente grueso y por algún concepto repugnante'. Foz (106) registraba como vulgar botinchado 'muy hinchado'. Moneva (106) recogía botincháu, da 'hinchado' (Litera), 'amostazado, amoscado' (Litera), 'de aspecto pretencioso habitual' (Zaragoza), 'enfadado contra otro por vanidad o soberbia' (Zaragoza), 'persona que se halla enfadada y hace el serio sin proferir queja' (Puebla de Híjar, Escatrón), 'hombre o animal que tiene el cuerpo inflamado por causa de alguna enfermedad, como la albuminuria', y botinfláu, da 'hinchado' (Zaragoza), 'persona muy gruesa, de aspecto repugnante' (Zaragoza). Pardo Asso (62) incluía en el mismo lema botinflado, da o botinchado, da 'hinchado como un boto', 'orgulloso, pedante, presuntuoso'. En Navarra, botinflado 'hinchado, abotagado', 'hombre desproporcionadamente grueso y por algún concepto repugnante', fig. 'orgulloso, pedante, presuntuoso, hueco' (Iribarren 89). Andolz (75) recoge botinchau, da y botinflau, da, procedentes de Pardo Asso, así como botiflón 'hinchado' (Bajo Aragón), y abotinchar-se 'hincharse, abotargarse', abotinchau, da

8. Eclesiástico del siglo XVII que escribió obras religiosas tanto en catalán como en castellano; según la Gran enciclopèdia catalana, era natural de la Cerdaña.

9. En su Colección de voces usadas en la Litera (1902) incluyó botinchado 'hinchado', 'amostazado, amoscado' (López Susín 8), y en su Colección de voces aragonesas presentada al Estudio de Filología de Aragón para su obra del Diccionario Aragonés. Tomo II (1921): botinchau, da 'el hombre o el animal que tiene el cuerpo extremadamente hinchado a consecuencia de alguna enfermedad' (Aliaga/Arnal 133).

10. En concreto, en la Colección de Jordana se lee: botinchado, da adj. "llámase así al que se halla enfadado y hace el serio sin proferir queja" (Aliaga 1999-2000, 354).

11. Ver el gascón boubat coum u bout 'soufflé (gonflé) comme une outre', "se dit d'un homme orgueilleux; d'un homme en grande colère" (Lespy/Raymond 123), oc. es coflat coma un bot 'c'est un gros vaniteux' (Lagarde 56), teniendo en cuenta que la persona orgullosa o encolerizada da la impresión de inflarse (de vanidad o de cólera). 
'hinchado' (Sarrión). En el valle de Gistau botinflau, -ada "se dice de la persona mal vestida o descuidada en la vestimenta" (Blas/Romanos 126).

Coromines (DECat II 171-72) 12 $^{2}$ registra el murciano botinchado, -inflado, que cree procedente del catalán botinflat, y el churro botinchado 'hinchado' (Titaguas).$^{13}$ Hay que añadir que continúa en otras hablas orientales peninsulares: en la Mancha, botibinchado, da "dícese de quien está excesivamente gordo, o bien padece hinchazón por herida o golpe o por efecto de algún humor" (Serna 75), en la Rioja, botinchón, na 'hinchado como una vejiga' y botincha 'vejiga llena de aire' (Pastor Blanco 100).

Sobre el origen etimológico, Peralta (XIII) consideraba el aragonés botinflado un compuesto "de boto (cuero) e inflar". Igualmente, en el Diccionario bistórico de la lengua española (1936) de la RAE, botinchado, da se analiza como formado "de boto e binchado" (NTLLE). ${ }^{14}$ Por su parte, Serna (75) cree que botibinchado se habría formado "de bota e binchar". Coromines (DECat II 171-72) piensa que el catalán botinflat parece ser un compuesto de bot-inflat o de botitinflat, ${ }^{15}$ y afirma que este tipo léxico "assolí ampla difusió dialectal en els parlars romànics de Catalunya, d'Occitània i d'algunes zones francoprovençals i italianes". Aunque Coromines no lo considera, pues solo cita el botinchado de Titaguas, tiene también, como vemos, una amplia vitalidad y extensión en tierras aragonesas y navarras, donde ha desarrollado diversos significados, con continuidad en otras hablas peninsulares orientales.

En el caso del catalán, el DCVB registra botinflar 'inflar excessivament' y botinflat 'inflat excessivament, deformat per l'inflor' en valenciano y tortosino, y la variante botunflat en el catalán de la Ribagorza. ${ }^{16}$ Coromines (DECat), siguiendo el Diccionari Aguiló, documenta botinflat por primera vez en catalán en la Rondalla de rondalles (1776) del valenciano Lluís Galiana, y afirma que es "més vivaç en els dialectes val. i occid., si bé no estrany als altres dialectes, especialment eivissenc", aquí alterado en putinflat. Ahora bien, de las hablas ca-

12. No lo registra en el DECH.

13. De hecho, en la zona castellanoaragonesa del interior valenciano tiene también gran extensión este tipo léxico (botinchao, botinchau, botinflao, botinflón, abotinflarse, abotinchase) (Gargallo 1987, 248-49). En valenciano también se documenta botinfló, análogo al churro botinflón: "VISANTET: -Per què-m tanques en l'armari, / botinflo? Encà se riu..." (Vidal i Roig 44).

14. Ver la comparación arag. más binchau que un boto (Jaime/Jaime 252).

15. Igualmente, en el DCVB se dice que botinflar es un compuesto de bot e inflar.

16. Con la cita de más arriba del periódico El Mole, vemos que botunflat también es conocido en valenciano. Se trata de una variante formada sobre unflat (unflar), variante de inflar, explicable por labialización de la $i$ inicial átona en contacto con el grupo labial [mf]. 
talanas noroccidentales solo se cita expresamente su presencia en tortosino, ${ }^{17}$ dialecto con notables coincidencias léxicas con el valenciano (lo mismo se puede decir del ibicenco), y en ribagorzano, donde los contactos con el aragonés son evidentes. Así, pues, independientemente de cuál sea la procedencia de boto en aragonés, ${ }^{18}$ el compuesto aragonés botinflado, botinchado posiblemente sería determinante en la distribución valenciana y tortosina de botinflat en catalán. De hecho, como hemos visto, incluso se documenta con anterioridad botinchado a la forma catalana correspondiente.

estrela / estrela

con estrelas en el cielo. (López Allué 336)

venerant al sol y a les estreles (Casanova 1988, 406); que pareix la lluna hermosa / tota de estreles cercada. (Coloqui de Pep... 90 $)^{19}$

Coromines (DECH II 805; DECat III 764) explica la $r$ del castellano estrella, del portugués estrela, y del dialecto italiano de Bolonia strela ( $<$ lat. STËLLA) por repercusión o anticipación de la otra líquida del vocablo. Ahora bien, en el caso del Alto Aragón, ${ }^{20}$ afirma que estrela es una "forma acatalanada". ${ }^{21}$ Sin embargo, hay que tener en cuenta que las formas patrimoniales y tradicionales en catalán son estel y estela, y que estrella y estrela son consideradas por el mismo Coromines como castellanismos, teniendo en cuenta que "no se hallan nunca en la Edad Media, y la primera contradice la norma fonética catalana de simplificación de la -LL- tras vocal larga" (DECH). En el DECat (III 764) parecería

17. Para el tortosino botinflat, ver Beltran i Cavaller (143).

18. El catalán bot 'odre, recipient de cuiro per al transport, sobretot del vi', como el occitano bot 'odre' y otras formas galorrománicas, el engadino buot y el italiano botte, serían descendientes del latín tardío BUTTIS 'tonel', 'odre' (DECH I 639; DECat II 151-52). En el DECat Coromines se refiere también a la presencia altoaragonesa de boto y botico 'odre de vino', en el Valle de Vió, así como a un mozárabe bote, boto, sin pronunciarse expresamente sobre un supuesto origen foráneo de la forma aragonesa, cosa que sí que hace en el DECH, donde afirma que el aragonés boto 'odre', botillo 'odre pequeño' se tomó del catalán, a diferencia del salmantino boto 'bota pequeña', que se habría formado a partir del castellano bota, "tal como canasto de canasta y casos análogos". Sobre el castellano bota 'vasija de cuero para beber vino', cree posible que proceda del catalán o del occitano, teniendo en cuenta su documentación más tardía, así como "su significado más restringido y menor vitalidad", si bien reconoce que "esta familia no es ajena al castellano".

19. Ya la documentamos en el siglo XVII: "que penetrà les estreles" (Sacro monte... 227).

20. Registra, asimismo, el masculino estrel en el este altoaragonés. Estrel se conoce también en el catalán de la Litera (Giralt 229).

21. Añade que "la forma etimológica estella se conservó antiguamente en Aragón, pues aparece todavía en el Cronicón Villarense de h. 1210 [...], como prolongación del cat. estela o estel". 
otorgar al valenciano estrela el carácter de voz genuina, cuando afirma que allí "la forma nacional amb $l$ simple ha estat augmentada amb la mateixa $r$ epentètica o repercussiva que el cast. estrella i el port. estrela", pero en otro lugar del mismo DECat (I 466) no niega la influencia del castellano estrella sobre el valenciano estrela: "la influència d'astre i la seva família $[. . .]^{22}$ degueren cooperar bastant, en certs medis cultes, amb el model del cast. estrella, en l'èxit que obtingué la variant estrela (val.), estrella (Princ.) en català modern”. Por su parte, Veny (PALDC, mapa 422 "Els estels") afirma que en catalán estrella es un préstamo del castellano, mientras que el valenciano estrela sería, según este filólogo, resultado de un cruce de estela con estrella.

Según Coromines, estrella, no recogida en catalán "fins als segles decadents [...], no s'afermà fins cap a 1800" (DECat III 762); en cuanto al val. estrela, el DECat no la documenta hasta 1934, y el DCVB solo la recoge en el poeta Teodor Llorente, bien avanzado el siglo XIX. En los textos valencianos que citamos más arriba se encuentra, como vemos, desde el último cuarto del siglo XVII. ${ }^{23}$ Además del valenciano, en catalán estrela es conocida en hablas del Bajo Aragón y del Bajo Cinca. ${ }^{24}$ En contraste con la presencia moderna de estrela en catalán, en cambio, estrela se documenta ya en textos aragoneses medievales (Fueros de Aragón 1237; Poesías de Pedro de Santafé, siglo XV. CORDE). Por tanto, teniendo en cuenta la extensión limitada y tardía de estrela en catalán, y su mayor extensión y antigüedad en aragonés, nos inclinamos más bien por un aragonesismo más del valenciano.

\section{a gatomiau / a la gatamau}

Dispués bajó a gatomiau las escaleras. (Botana, serie segunda 56)

M'amague darrere un chop, / y d'allí, a la gatamau, / em despeñe presurós / en una séquiaprofunda. (Un pillo... 46)

La locución adverbial a gatomiau no se registra en el DRAE ni en el DECH. Sin embargo, se conoce en Aragón: a gatomiau 'a gatas' (Sánchez/Tomás 208), an-

22. En cambio, en el DECH (II 805) niega la influencia de ASTRUM en la formación de la $r$ de este tipo léxico.

23. El poeta renacentista valenciano Joan Antoni Almela, en un poema culto de 1855 lleno de arcaísmos, usó la voz tradicional catalana estela, pero con la glosa estrela, que correspondía a la forma popular en el valenciano de mediados del siglo XIX: "mes una estela (estrela) per l'orient asoma" (Fiestas que en el siglo IV... 316).

24. En el DCVB se registra estrela en valenciano y en la localidad aragonesa de Fraga (Bajo Cinca); el PALDC (mapa 422 "Els estels") la recoge también en la Codoñera (Bajo Aragón). 
dar a gatomiau 'andar a cuatro pies' (Moneva 259), y en Navarra: andar a gatomiau 'a cuatro manos' (Iribarren 258). En catalán, a la gatamau y a gatameu se registran en valenciano, ${ }^{25}$ y a gatameus 'de quatre grapes' en Tortosa (DCVB, DECat IV 379, 419); en Burriana (Castellón), a gatomiau (Roselló), como en aragonés. Voz compuesta de gato o gata y de la voz onomatopéyica meu, mau o miau, imitativa de la voz del gato.

moñica, muñica 'muñeca de la mano' / monyica, munyica

un reloj en la moñica / atau con una correa. (López Allué 240)

a la monyica també / del braç un troset de grana, / o una figueta, perquè / no prenguen l'infantet d'ull (Romanç nou, molt graciós... 3); torna a tòrser la muñica. (El Sueco 198)

En castellano las formas con la terminación -ica, muñica y moñica, no se registran en el NTLLE, ni en el DECH, ni tampoco se encuentran en la base de datos CORDE, pero sí que se hallan en diccionarios aragoneses: moñica 'muñeca de la mano' (Agliberto Garcés 235; Andolz 297) y muñica (Nagore 1999, 1316; Rodés/Maza/Gavín 135; Mostolay 392).

En catalán se registra munyica en valenciano y tortosino, y monyica en valenciano (DCVB). Según el DCVB, procedería del castellano muñeca, y Coromines (DECat V 781) plantea la posibilidad que el valenciano munyica sea un mozarabismo. Sin embargo, lo más probable es que haya llegado al valenciano desde el aragonés.

\section{Palabras de uso en Aragón, no Registradas hasta AHORA EN VALENCIANO}

coscarana / coscarrana

Debías de dale güenas plateradas de sopas de pan ú coscarana, pa que no lapetecieran tanto los pechos. (Botana, serie primera 84 )

Si empanada [...] / ha tengut ta camarada, / per Déu, sou afortunats, / y més si ha agut botifarra. / A mi no me aplega un llam, / més que alguna coscarrana. ${ }^{26}$ (año 1779) (Martí 2011, 60)

25. Por extensión, en valenciano, gatameus 'bebé': "El tio Pep acamina / cabilós [...], / perquè no troba l'estrel / que.l guie cap a la dona / i cap al seu gatameus" (Falla ... 13).

26. Referencia al hambre que pasa. 
El sustantivo coscarana 'torta muy delgada y seca que al comerse cruxe' figura en el DRAE desde la edición de 1780, como voz usada en Aragón. Con anterioridad se registra en el Borrador de un diccionario de voces aragonesas, elaborado entre 1715 y 1724 por Siesso de Bolea: coscarana 'torta delgada y seca con arina dentro'; el mismo autor plantea una curiosa explicación etimológica, como un compuesto "de casca y arina, casca arina" (Aliaga 2008, 131). ${ }^{27}$ En Aragón coscarana ha adquirido otros significados relacionados, que designan objetos duros, endurecidos o secos, o incluso golpes: 'caparazón', 'esqueleto', 'corteza del árbol', 'tastarrazo que le cae a uno' (Nagore 1999, 599), 'costra que se levanta en paredes, piel de personas, etc.' (Negredo 103), 'costra seca exterior, que se desprende o rompe fácilmente' (Mercadal 56), 'cáscaras' (Castro 10), 'farfolla' (Garcés 1989, 104). En los Monegros, la coscarana es una "torta delgada en forma circular y cóncava”, y figuradamente, "cuando la tierra se reseca y resquebraja, formando costras, se dice que la tierra se ha puesto como una coscarana" (Rodés/Maza/Gavín 83). También se conoce en la región la variante cascarana. Así, en Sarrión conviven con el mismo sentido coscarana y cascarana (Andolz 106, 124), como en el Somontano de Barbastro, donde la cascarana es una "pequeña torta, fina y crujiente, que lleva como ingredientes: agua, harina, media cáscara de azúcar, media cáscara de aceite y media cáscara de anís dulce (de ahí el nombre de cascarana)" (Mostolay 150). En Benasque la cascarana es la calavera, conocida como coscarina en la localidad altorribagorzana de Ardanuy, de habla ya catalana (Nagore 1999, 458). En el romance de Navarra se conocen las variantes coscaraña, cascaraña, cascarraña, tostaraña y testaraña. Según Iribarren (118), “cascarraña es voz onomatopéyica que imita al crujido del pan al partirlo o comerlo". En efecto, Kruger situaba también el aragonés coscarana entre las voces formadas con la raíz onomatopéyica $\operatorname{cosc}-$, del mismo tipo que casc-, coc- y croc-. Designarían de forma imitativa el ruido de un golpe o choque, así como objetos duros o secos, que producen un sonido característico cuando se rompen, se golpean o se muerden. Una explicación similar se encuentra en el DECH (II 221).

En contraste con la rica presencia aragonesa, en cambio, en catalán los testimonios de este tipo léxico son muy escasos, y de carácter dialectal, en dia-

27. En el mismo diccionario vemos que la casca designa "la cáscara. Y el cascarón del huevo" y el "roscón de manzana y alcorza", por "la casca que cubre el mazapán y también de estar cascadas y quebrantadas las cosas que entran dentro". 
lectos donde la influencia aragonesa es indudable. Quintana (100) recogía en la localidad bajoaragonesa de la Codoñera la forma coscarana 'coca rodona amb ous, oli escaldat i sucre', y hemos visto más arriba la presencia de coscari$n a$ en la Alta Ribagorza de lengua catalana. En valenciano, fuera del citado texto del siglo XVIII, no encontramos otros testimonios de esta voz. Aunque en catalán no son desconocidas las palabras formadas con las raíces onomatopéyicas cosc- y casc-, como coscorró, coscorro, cascarró, cascarro, cascarrós, cascarra, cascarrar, cascàrria o casca, y esta última, además del sentido de 'closca, clovella dura', tiene también dialectalmente el de 'espècie de coca circular foradada en el centre, feta de pasta ensucrada i untada' (DCVB) ${ }^{28}$ dadas las circunstancias comentadas, el caso de coscarrana debe de ser un aragonesismo del valenciano. Esta forma pudo llegar directamente de tierras aragonesas, donde, aunque predominan las formas con $r$ en este tipo léxico, tampoco parece ser desconocida la forma coscarrana: "coscarranas con nueces garrapiñadas" (ver Martí 2011, 77), y además recordemos las formas navarras también con $r$. Ahora bien, también podría tratarse de una adaptación a partir del aragonés coscarana, teniendo en cuenta el uso más destacado en catalán de las palabras con $r r$ en las voces formadas con cosc- y casc- y la relativa facilidad del intercambio entre las vibrantes. ${ }^{29}$

\section{tasamente / a tassament}

Al verme tan esturdido / endrecé cara pal cuarto / y tasamente podía / amparame en los peldaños. (López Allué 168)

CARMELO: -Vol ohuir-mos un instant? / MATEU: -No cal, perqu $\cdot$ a tasament / sobre lo que.m vas a dir... / CARMELO: -Com!... Sap?... (Balader 39); -Fan dubtar a cuansevol que les vecha, y per a casar-se una chica, no és menester arribar a eixe punt. $-A$ tasament, a ella li plouen els nóvios, y més qu·en volguera! (Foc y flama 2)

En aragonés se conoce el adverbio tasamente 'escasamente, tan solo, apenas', con las variantes tasamén, tásamen y tansamén (Nagore 1999, 1740-41;

28. Colomina (171) relaciona el verbo cuscarrar 'insistir', usado por el escritor valenciano Enric Valor, con las voces tortosinas cascurrer 'pegador, que té propensió a maçolar' y cascurrejar 'pegar, ferir amb la mà pel cap o per la cara', que, según el DCVB, derivan de la misma raíz de cascarro y cascar.

29. Sobre el paso de vibrante simple a múltiple y de vibrante múltiple a simple en catalán, ver Martines (225-40). 
Nagore 2000, 569; Andolz 409; Romanos Hernando 241; Rodés/Maza/Gavín 164; Eito 157; Mostolay 519; Blas/Romanos 486). El adverbio tasamente se extiende por Castilla-La Mancha (Cuenca, Albacete, Ciudad Real, Toledo) (Mora Rodenas; Díaz Buenestado; Sánchez Miguel) y llega a tierras andaluzas, a las provincias de Almería y Jaén: tasamente 'tasadamente, escasamente', 'poca cosa, casi, casi' (Alvar Ezquerra 757). Por otra parte, Agliberto Garcés (314) recogía en la comarca oscense de Bolea tasamente con el sentido de 'precisamente'. Encontramos este mismo significado en las hablas castellanoaragonesas del País Valenciano: tasamente 'precisamente' (Llatas 193; Nebot Calpe 519; Gargallo 2004, 101), ejemplo: "Vino cuando tasamente m'iba a dormir" (Llatas). Se trata de una solución fonética diferente a la que muestra el castellano tasadamente (ant. tassadamente), que recogía el Diccionario de Autoridades (1739), con el sentido de 'con medida o tassa, limitada y escasamente', ${ }^{30}$ y que luego pasa al DRAE. El aragonés Siesso de Bolea registraba también en Aragón, en el primer cuarto del siglo XVIII, la forma tassadamente: "Quando negamos algo solemos decir «tassadamente!», como quien dice, por ironía, «sí, esto es». En Castilla propiamente! (creo)". La información de este autor sobre tassadamente da a entender un significado muy próximo a 'precisamente' o 'propiamente', si bien usado irónicamente, lo cual lo aproxima mucho al valor que indicaba Garcés y que hemos visto en las hablas castellanoaragonesas valencianas. De hecho, teniendo en cuenta el significado de tasar 'fijar', 'graduar', 'restringir', se entienden los diversos sentidos del derivado tasadamente (y aragonés tasamente), que pueden indicar tanto la idea de escasez o limitación como la de precisión.

En valenciano documentamos la locución adverbial a tassament en la segunda mitad del siglo XIX y en el primer tercio del siglo XX, con el sentido de 'precisamente, propiamente'. La falta de testimonios en otros dialectos catalanes da a entender que debió de llegar al valenciano a través del aragonés. La presencia del castellanismo tasa 'taxa' en valenciano desde el siglo XIX favoreció el préstamo. ${ }^{31}$

30. Con anterioridad se encuentra en diccionarios del siglo XVII, y ya desde finales del siglo XVI (NTLE).

31. En valenciano parece ser que también se introdujo la forma tasadament 'tasadamente: con medida o tasa', familiarmente 'cabalmente, precisamente', que se registra en Martí Gadea, si bien la forma que encontramos más habitualmente en los textos popularistas de la época es a tasament. 
ARCAÍSMOS ESPAÑOLES CONSERVADOS EN ARAGÓN DESDE DONDE LLEGARÍAN AL CATALÁN DE VALENCIA

cutio, cutia, cutiamente / cútio, cútia

¡De cuántas cosas semos iznorantes los que siempre estamos cutios en los lugares! (Botana, serie primera 73); El desdichado viejo tuvo que pagar too y la madre, como resultancias de eso, comenzó con una cavilación cútia, cútia (Botana, serie segunda 24); ¿Y los mozos? Too era mirala cútio, cútio y se quedaban encantaus con ella (Botana, serie segunda 30); Por quererte $c u$ tio, cutio / me plantastes en la calle (García-Arista 35); ${ }^{32}$ aunque les dieran crestón / nunca se veían fartos, / pero tranquilos y cutios en lo de mover los brazos (López Allué 327); deja el porrón en la mesa / cutio, cutio y sin chistar, / se va al Gobierno civil / a quejarse al general (López Allué 342); ¡Barajo que criaturicas! Cuasi too el auto é la ciremonia me las estuve mirando cutiamente y aun así no ma contentaba é velas. (Botana, serie cuarta 85)

este és un temps en què tots / pasem el dia en lo camp. / Sols se enrecordem de Déu, / de lo demés enchamay, / només cútio a la faena, / a guañar un tros de pa (Branchat 56); Cútio había de estar sempre, / en aire, fret o calor, / al costat de aquell tirano / de cheni el més regañó ( $U n$ pillo... 29); Sols cuant algo té romput / u està mal sóc bona yo! / Y és que cútia al seu capsal, / fent tot cuant mana el dotor, / pa ell sa mare és la millor / hermana de l'hospital (Balader 13); MASCLET: -Ha vist pasar al siñor Melito? El CurRo: -Home, per casolitat no ha vengut hui. Perqu.ell és molt cútio! Lo que és el segon dia de mes a cobrar la paga de l'atre, no falta may. (La Traca núm. 56, 2)

En castellano antiguo era conocido cutio; según Coromines (DECH I 225), derivado regresivo del antiguo cutiano 'cotidiano, todos los días', variante semipopular de cotidiano (del latín QUOTIDIANUS). El mismo autor informa que era empleado en la expresión día de cutio 'día laborable' todavía por Cervantes. Alonso (842) documenta en español medieval cutio, en la locución de cutio 'de cada día', juntamente con el adjetivo cutiano, -na 'cotidiano' y el adverbio $c u$ tianamente 'cotidianamente, a diario', con las variantes cutianamiente y cutianamientre. Covarrubias (1611) registraba día de cutio "el de trabajar la gente, como son los días de entre semana que no son fiestas de guardar", como Fran-

32. En una nota del editor a pie de página se lee el sentido de cutio, cutio: 'con constancia'. 
ciosini (1620) y Trognesius (1639), y a principios del siglo XVIII, Stevens (1706) recogía dia cutio 'a working day, that is neither sunday, nor holiday', pero lo consideraba ya obsoleto (NTLE). En el Diccionario de Autoridades (1729) se define cutio como 'el trabajo material', haciendo una interpretación errónea de la expresión cervantina dia de cutio (ver DECH II 225), lo cual prueba el carácter anticuado que tenía ya entonces cutio en español, y así figura expresamente, como anticuado, en la edición del DRAE de 1780. En esta edición se incluye también la expresión trabajo cutio 'trabajo cortísimo', calificada de aragonesa, la cual desapareció a partir de la edición de 1817. Igualmente, en la edición de 1780 del DRAE se leen el adjetivo cutiano, na, sinónimo de quotidiano, y el adverbio cutiano "lo mismo que quotidianamente", calificados también de anticuados en la misma obra, los cuales desaparecerán del diccionario académico en la edición de 1822. En las ediciones de 1832, 1837, 1843 y 1852 se acentúa sobre la $i$, cutío. Salvá3 ${ }^{33}$ repite la definición errónea del DRAE sobre el sustantivo cutio, pero, conocedor del valor que tenía en valenciano, añade el uso del adjetivo cutio, -tia 'continuo', que califica de familiar, y que sería real en el valenciano de su tiempo. No será hasta la edición de 1956 que se cambiará y corregirá la presentación de esta palabra en el DRAE. Ahora será incluida como un adverbio, y no como un sustantivo: cutio adv. 'continuamente, seguidamente', de cutio loc. adv. 'de continuo, de asiento', y día de cutio 'día de trabajo'; además se añade el adjetivo cutiano, na 'diario, continuo', como antiguo y conservado en Aragón, ${ }^{34}$ así como el adverbio cutiano 'diariamente, continuadamente' y las locuciones adverbiales de cutiano y en cutiano. En la edición de 1992 del DRAE se informa que el adverbio cutio y la locución de cutio son desusadas en español general, y solo conservadas dialectalmente.

Cuando en español se veía ya como arcaico, en el primer cuarto del siglo XVIII, Siesso de Bolea lo recogía en aragonés: cutio 'continuo', con el ejemplo "Si estuviesse cutio en el travajo", y cuti, cuti 'de contado, continuamente, sin çesar' (Aliaga 2008, 135). En la actualidad cutio se conserva en Aragón, donde ha desarrollado distintos matices semánticos, como adjetivo y como adverbio. Como adjetivo, 'constante', 'fijo, permanente', 'atento, prestando atención a lo que se hace', 'que se esconde cuidadosamente, pretendiendo pasar desapercibido', 'escondido, disimulado', 'callado, silencioso', 'quieto', y adverbio 'sin

33. En el Nuevo diccionario de la lengua castellana, 1846 (información que citamos a través del NTLLE).

34. En la edición de 2001 se suprime el uso aragonés de cutiano. 
interrupción, seguido', 'siempre, para siempre', 'en silencio y despacio', 'lentamente', 'a escondidas, disimuladamente'; cutio cutio locución adverbial 'despacio, poco a poco', 'con cuidado', 'constantemente, sin interrupción', 'atentamente, con atención, pendiente de algo', 'a escondidas, silenciosamente, calladamente, con sigilo'; cutiano 'cotidiano' (Nagore 1999, 642; Negredo 104; Agliberto Garcés 122; Rodés/Maza/Gavín 86; Mostolay 197; Blas/Romanos 183); pa cutio 'para siempre', 'continuo, seguido, sin cambio, siempre igual' (Mercadal 60; Rodés/Maza/Gavín 86). También se conoce en Navarra: cutio 'constante; sin interrupción', cutiano 'cotidiano', 'constante, frecuente' (Iribarren 162), el noroeste murciano: cutio 'continuo, seguido, constante' (Ruiz Marín 221) y el nordeste de Andalucía: cutio, -tia 'que está a pie quieto, sin desplazarse o moverse, o sin actuar', estar de cutio 'estar fijo en un sitio, permanecer en algún lugar' (Alvar Ezquerra 298; Escobedo 74).35

En catalán, donde encontramos en los textos costumbristas citados más arriba la primera documentación conocida del vocablo, el DCVB registra cútio 'perseverant, constant' en valenciano y en la localidad bajoaragonesa de Calaceite, ${ }^{36}$ pero sin relacionarlo con el aragonés. En el DECat (VI 976) se recogen otros significados de cútio en valenciano: 'cotidiano', 'perenne', ${ }^{37}$ 'capficat i minuciós en les coses' (Benasal), 'contador dels dinerets i agarrat, interessat' (Maestrazgo), 'home repretat, tapat, que no ix de casa' (Morella). En el DECat parece considerarse el catalán cútio como un derivado de cutià, como el aragonés y castellano antiguo cutio serían derivados regresivos de cutiano. Ahora bien, hay que tener en cuenta que, a diferencia del español, donde cutio es ya medieval, en catalán cútio no se documenta hasta principios del siglo XIX, y que su uso se restringe al valenciano y a las hablas catalanas bajoaragonesas y del Bajo Cinca, y que, además, así como cutiano es ya medieval en español, no tenemos datos documentales de un supuesto cutià catalán. Por tanto, hay que pensar que en estos dialectos catalanes llega a través del aragonés, donde, como hemos visto, ha quedado como un arcaísmo que antiguamente tenía una mayor extensión en español. Casanova $(2003,197)$ ya decía que cútio "en va-

35. Según Escobedo (74), estar de cutio, pero según Alcalá Venceslada, estar de cutío, con acento sobre la $i$ (Alvar Ezquerra 298). En el DECH (II 225) se dice que la acentuación cutío de García de Diego es arbitraria, por entenderlo erróneamente como descendiente de quotidio.

36. También se conoce en la Codoñera, localidad, como Calaceite, del Bajo Aragón catalanohablante: cútio 'persona que treballa sense parar i en silenci', cútio, cutiet 'sense parlar' (Quintana 108, 112). En Fraga, cútio, -tia 'tímido' (Galán Castañ/Galán Pérez 17).

37. Estas dos acepciones proceden de Escrig, que recoge también la variante cuti, y el adverbio cútiament 'cotidianamente', 'perennemente o perennalmente'. 
lencià és un dels mots introduïts pels parlars xurros o aragonesos, que només viu en les zones de contacte més estret". Si bien compartimos substancialmente la opinión sobre el origen aragonés de esta voz, creemos que más bien sería introducida directamente por contacto con población aragonesa, y no tanto desde las hablas valencianas de base aragonesa, teniendo en cuenta que no parece ser especialmente conocida en estas comarcas, ${ }^{38} \mathrm{y}$ en cuanto al uso en valenciano, este, al menos en el siglo XIX, no se limitaría a las zonas de contacto más estrecho con el aragonés, sino que, como vemos a través de los textos procedentes de la zona central valenciana, debía tener un uso bastante generalizado en esta zona. ${ }^{39}$

\section{PALABRAS ARAGONESAS Y VALENCIANAS DE DOCUMENTACIÓN MODERNA QUE EN CATALÁN PARECEN NO LIMITARSE AL USO VALENCIANO, PERO EN LAS CUALES LA INFLUENCIA ARAGONESA DEBIÓ DE SER DETERMINANTE PARA SU PRESENCIA DESTACADA EN VALENCIANO}

\section{acabacasas / acabacases}

Algunos ya podrán acudir con el buche lleno, pero lo ques otros, pue que vengan con tres cuartos de hambre y cinco de nesecidá. De muchismos dellos se pue icir que son unos acaba-casas" (Botana, serie cuarta 69). ${ }^{40}$

Un chermà que va fent l'ensa, / convoyat per un parent / qu.és un vago acabacases" (Alberola 42); Y en dos mesos has gastat ya dos pesetes?... Malo, malo, malo!... Tu vas camí d’acabacases. (Hernández Casajuana 3)

La palabra compuesta acabacasas no se encuentra en el NTLLE, en el NTLE, en el DECH, en la base de datos CORDE, ni en el Corpus del español de Davies. La recogen, sin embargo, diversos diccionarios y repertorios léxicos aragoneses. Coll y Altabás (1902) incluía acabacasas 'dilapidador, manirroto' entre las voces de la Litera (López Susín 1). Moneva, hacia el año 1924, registraba acabacasas en la Ribagorza. Pero tiene una mayor extensión en Aragón, como muestran las recopilaciones más recientes. Se encuentra en el Alto Aragón (Valle de Gistau, Somontano de Barbastro, Cinco Villas, Jacetania) (Nagore 1999, 83;

38. No la recogen Rojas Clemente (Martín/Tello), Torres Fornes, Monzó Nogués, Llatas, Alba, Martí/Aparicio, Gargallo ni Doménech.

39. Aún hoy no es desconocido en las comarcas centrales valencianas. En el PALDC (mapa 505 "Treballar a preu fet") se registra cútio en la localidad valenciana de Sollana.

40. Por referencia a los estudiantes. 
Eito 45; $;^{41}$ Blas/Romanos 61), se extiende a los Monegros, acabacasas 'persona derrochadora y poco trabajadora' (Rodés/Maza/Gavín 42), al Bajo Cinca (Soler Betés 65), a Zaragoza (Gargallo Sanjoaquín 13) y llega hasta la provincia de Teruel (Mercadal 12), a la vecina de Guadalajara (Marco Yagüe 22), así como a las hablas valencianas castellanoaragonesas (Llatas 89; Martí/Aparicio 12). Se conoce también en el romance de Navarra (Iribarren 25).

En catalán, además de los testimonios valencianos, acabacases se conoce en la comarca noroccidental extrema de la Litera (Giralt 56), pero, aunque no se encuentra en el DIEC, no parece ser un término limitado a estos dialectos, que pudiera, por esto mismo, explicarse con relativa facilidad como un aragonesismo. En el DCVB se documenta en el barcelonés Miquel Llor (1950), ${ }^{42}$ y Dorca (151) da testimonio de su presencia en localidades de la Selva y de la Plana de Vic, lo cual da a entender una mayor extensión en esta lengua. Así pues, no resulta tan sencillo pronunciarse sobre su posible origen aragonés, catalán o surgido en ambas variedades lingüísticas. La ausencia de testimonios antiguos no favorece tampoco esta labor. ${ }^{43}$ Con todo, parece tener una mayor extensión y vitalidad en valenciano, probablemente junto al ribagorzano, que en otros dialectos catalanes. De hecho, si bien no lo recoge el DIEC, sí que aparece en el DNV y ha desarrollado en valenciano otras acepciones. ${ }^{44}$ Por ello, no parece fuera de lugar pensar que su presencia más visible en este dialecto, como también en la Litera, esté favorecida por el aragonés.

41. La definición de Eito resulta especialmente transparente para entender la formación del compuesto: acabacasas 'persona que con su mal comportamiento, o por su mala gestión, pone en peligro la supervivencia de la casa'.

42. En el DCVB se documenta solo en este autor; por tanto, los testimonios valencianos que citamos más arriba representan la primera documentación conocida de la palabra en catalán.

43. Según Coromines (DECat II 514), que se limita a recoger el compuesto acabacases sin más información sobre su origen, extensión o documentación, el verbo acabar procede del latín vulgar *ACCAPARE, creación propia del románico primitivo hispanogálico, derivado del latín vulgar CAPU (por CAPUT). En francés antiguo achever tenia el sentido figurado de 'ruiner la fortune (de quelqu'un)' (Rey 26); en occitano acabar 'dissiper son bien', y el derivado acabaire 'celui qui achève, dissipateur, prodigue' (Alibert 69), en gascón también se conocen desacabar 'ruiner complètement', desacabayre 'dépensier sans mesure' y el nombre compuesto acabe-tout 'prodigue, dissipateur' (Palay 317), análogo a nuestros acabacasas, acabacases.

44. En el Ráfol de Almunia (la Marina Alta) (a)cabacases es el nombre popular del Centaurium quadrifolium (L.), subespecie linariifolium (Lam.), según parece, por las ganas de comer tan grandes que da esta planta (Pellicer 66). Por otra parte, en valenciano, en la primera mitad del siglo XX, se llamaba acabacases (y cabacases) un jarabe hecho a base de alcohol y hierbas medicinales, al que se atribuían virtudes curativas, pero que, como dice Llorente Falcó (III 285), en realidad, "no era más que un buen sudorífico que iba muy bien para las dolencias del aparato respiratorio y nada más". Parece ser que era fabricado y distribuido por el valenciano Vicente Cutanda Ros, conocido como Cabacases, como se lee en un libro de falla de 1931. 
carasol / cara-sol

fa una miqueta de cierzo, pero aquí en lo carasol no fa mal orache. (Miral 34)

em pinten [...], / puchant-me a este cara-sol, / fins a que se hacha secat / lo vestit nou ( $E l$ pardal... 4); Pos avans que els polls y la fam mos traguen a ballar a un cara-sol, vestim-os de botarga y ballem. (El Mole 1837, 105-06) ${ }^{45}$

Se extiende, según Coromines (DECat VIII 18-19), ${ }^{46}$ por "la franja or. del domini castellà", en concreto lo registra en La Mancha, y llega al gascón. Podemos añadir que es bien conocido en Navarra y Aragón (Iribarren 113; Moneva 138; Andolz 100; Blas/Romanos 149; Nagore 1999, 436), donde ya lo recogía Siesso de Bolea en el primer cuarto del siglo XVIII, por oposición a la voz castellana solana: carasol "el lugar donde da el sol de cara. En Castilla, sola$n a$ ". En catalán parece ser utilizado sobre todo en valenciano y tortosino (ver DECat; Beltran i Cavaller 195), si bien hay también noticias de cara-sol en las hablas occidentales de la Litera (Giralt 122) y del Alto Urgel (aquí como adjetivo) (DECat), así como en la Cerdaña, ya en catalán oriental (DCVB). Como parece ser una palabra de documentación moderna, tanto en catalán como en aragonés, no es fácil dilucidar si en valenciano ha llegado desde el aragonés o desde el catalán de los pobladores, o desde ambos grupos, si bien el uso aragonés ha debido de favorer su vitalidad en valenciano, ${ }^{47}$ donde lo documentamos desde el último cuarto del siglo XVII.

\section{CONCLUSIONES}

En el terreno del contacto entre el aragonés y el valenciano es recomendable la prudencia a la hora de pronunciarse sobre el posible origen aragonés, catalán o común de un determinado fenómeno, teniendo en cuenta la contigüidad geográfica de ambas variedades lingüísticas y las lagunas documentales que todavía existen sobre muchos fenómenos. Con todo, el estudio léxico comparado entre ellas se manifiesta, como hemos visto, muy productivo.

45. En valenciano ya se documenta en el siglo XVII: "Haveu vist un pobret ple de plagues, que està a un cara-sol, a hon se'l menjen les mosques?" (Mercader 1677, 368).

46. El DECH (I 841) se limita a incluirlo entre los compuestos de cara, sin más información.

47. Se encuentra también en algunas hablas castellanoaragonesas del País Valenciano, como la Canal de Navarrés (Martí/Aparicio 38) y los Serranos (http://lenguachurra.blogspot.com.es/2012 /01/parque-natural-chera-sote.html). 
El análisis de una muestra representativa de formas léxicas procedentes de textos costumbristas de los siglos XVIII, XIX y XX permite aportar nuevos datos y correcciones a la bibliografía existente. Este estudio nos ha permitido encontrar: voces valencianas no registradas en el DCVB ni en el DECat, primeras documentaciones de las voces valencianas y vocablos de uso en Aragón no registrados en el DECH, o registrados pero no en este territorio.

Hemos dividido el trabajo en diferentes apartados, que representan diversas posibilidades en la relación histórica entre el valenciano y el aragonés.

Este planteamiento nos permite concluir que las obras lexicográficas no siempre han tenido en cuenta la relación entre las formas aragonesas y las valencianas, así como la posible influencia de las primeras sobre las segundas. De los resultados de nuestro trabajo se desprende que para estudiar muchas de las particularidades léxicas del valenciano hay que considerar las formas aragonesas y su continuidad en otras hablas orientales peninsulares.

Algunas de las voces valencianas explicadas tradicionalmente como castellanismos o mozarabismos puede que sean más bien aragonesismos, si tenemos en cuenta la forma de las palabras, la fecha de documentación y su presencia sobre todo en tierras aragonesas (bolchaca / bolxaca; moñica, muñica / monyica, munyica; estrela).

En ocasiones, los diccionarios no han puesto en relación las formas aragonesas con las palabras catalanas, a veces porque no registran determinadas voces españolas de uso en Aragón (a gatomiau, acabacasas). Si las relacionamos, podemos apreciar de forma más plena que el léxico peninsular oriental a veces sobrepasa los límites lingüísticos españoles y se encuentra también en catalán, especialmente en valenciano.

Por otra parte, la explicación de ciertas voces como catalanismos del español, por su uso en murciano y en las hablas valencianas de base castellana, quizá se podría relativizar si se tuviese en cuenta su mayor extensión en Aragón y en el español oriental así como su localización sobre todo valenciana en catalán (botinchado, botinflado / botinflat, botunflat).

Se recogen también palabras no registradas hasta ahora en valenciano, de uso extenso en Aragón, y de escasa presencia catalana, y esta en dialectos próximos a las tierras aragonesas (coscarana / coscarrana, tasamente / a tassament).

Otro apartado de la relación del valenciano con el habla de Aragón es el de los arcaísmos españoles conservados en este territorio, desde donde llegarían al catalán de Valencia (cutio / cútio). 
Por último, en algunas voces con manifestación destacada en valenciano, pero no limitadas a este dialecto catalán, la presencia aragonesa pudo ser determinante (acabacasas / acabacases, carasol / cara-sol).

\section{OBRAS CITADAS}

Alba, Isabel. El habla de Ludiente. Castellón: Diputació de Castelló, 1986.

Alberola, Estanislau. Terres secanes. Valencia: José Olmos, 1924.

Aliaga, José Luis. "Documentos lexicográficos del Estudio de Filología de Aragón (II)". Archivo de Filología Aragonesa 56 (1999-2000): 337-442.

Aliaga, José Luis. Borrador de un diccionario de voces aragonesas: Fosé Siesso de Bolea. Zaragoza: Gara d'Edizions, 2008.

Aliaga, José Luis, y María Luisa Arnal. Textos lexicográficos aragoneses de Benito Coll (1902-1903) presentados al Estudio de Filología de Aragón: edición y estudio. Zaragoza: Pórtico, 1999.

Alibert, Louis. Dictionnaire occitan-français. Toulouse: Institut d'Estudis Occitans, 2002.

Alonso, Martín. Diccionario medieval español. Salamanca: Universidad Pontificia de Salamanca, 1986.

Altaba Escorihuela, José. Teruel: peculiaridades de nuestro léxico popular (Más de tres mil palabras de uso popular regionalista). Zaragoza: Librería General, 1985.

Alvar, Manuel. El dialecto aragonés. Madrid: Gredos, 1953.

Alvar Ezquerra, Manuel. Tesoro léxico de las hablas andaluzas. Madrid: Arco Libros, 2000.

Andolz, Rafael. Diccionario aragonés. Zaragoza: Mira, 2004.

Balader, Joaquim. Eixarop de llarga vida. Valencia: Ferrer de Orga, 1863.

Beltran i Cavaller, Joan S. Vocabulari de cruilla. Benicarló: Onada, 2010.

Blas Gabarda, Fernando, y Fernando Romanos Hernando. Fraseología en chistabin. Zaragoza: Gara d'Edizions/Institución Fernando el Católico, 2008.

Borao, Gerónimo. Diccionario de voces aragonesas. Zaragoza: Ariño, 1859.

Botana, Crispín. La gente de mi tierra en las fiestas del Pilar de Zaragoza. 6 series. Serie primera. 2. ${ }^{a}$ ed. Zaragoza: tip. de Comas, 1900; Serie segunda. 3. ${ }^{a} \mathrm{ed}$. Zaragoza: tip. Andrés Arantegui, 1904; Serie cuarta. 2. ${ }^{\text {a }}$ ed. Zaragoza: tip. de Comas, 1896; Serie quinta. 2. ${ }^{a}$ ed. Zaragoza: tip. de Comas, 1897.

Branchat, Vicent. Conversasió que tingueren el tio Bernat, ortolà de València, en lo seu hort, y Miquelo el Tosut del lloch de Benaguacil, sobre les notícies que han co- 
rregut y corren de Malapart. Ms. 661. Valencia: Biblioteca Universitària de València, 1815.

Casanova, Emili. "Sobre els sermons de les festes de Sant Vicent Ferrer al segle XVIII a València". Miscelllània d'homenatge a Enric Moreu-Rey. Vol. 1. Eds. Albert Manent y Joan Veny. Barcelona: PAM, 1988. 385-407.

Casanova, Emili. "Què té el valencià d'aragonés? Particularitats del valencià a causa de l'aragonés". Actas del V Congreso Internacional de Historia de la lengua española. Eds. María Teresa Echenique y Juan Pedro Sánchez Méndez. Madrid: Gredos, 2002. 1661-77.

Casanova, Emili. "El lèxic de la decadència en els estudis etimològics: el cas del DECat de Joan Coromines". Actes del dotzè Col.loqui Internacional de Llengua i Literatura Catalanes. Eds. Marie-Claire Zimmermann y Anne Charlon. Barcelona: PAM, 2003. 189-221.

Castro Merino, Andrés. "Lesico de Billar d'o Salz". Ruxiada 10 (1992): 6-21. Colomina, Jordi. "El valencià popular i col-loquial en l'obra narrativa d'Enric Valor". Valoriana: estudis sobre l'obra d'Enric Valor. Eds. Vicent Salvador y Heike van Lawick. Castellón: Universitat Jaume I, 1999. 165-78.

Coloqui de Pep el de la Cascabellada, fill de Alboraya. Ms 419. Valencia: Biblioteca Valenciana. 90-105.

CORDE: Real Academia Española. Banco de datos [en línea]. Corpus diacrónico del español. <http://www.rae.es> [enero de 2017].

Davies, Mark. Corpus del español. <http://www.corpusdelespanol.org/histgen/2008/x.asp> [enero de 2017].

DCVB: Antoni Maria Alcover y Francesc de B. Moll. Diccionari Català-ValenciàBalear. 10 vols. Palma de Mallorca: Moll, 1988.

DECat: Joan Coromines. Diccionari etimològic i complementari de la llengua catalana. 10 vols. Barcelona: Curial, 1988-2001.

DECH: Joan Coromines, con la colaboración de José A. Pascual. Diccionario crítico etimológico castellano e hispánico. 6 vols. Madrid: Gredos, 1980-1991.

Díaz Buenestado, Francisco. Vocabulario Cucón. Vocabulario de Fuencaliente. $<$ http://fuencaliente.net/vocabulario_archivos/t.htm> [enero de 2017].

Diccionari Aguiló: materials lexicogràfics aplegats per Marian Aguiló i Fuster. Eds. Pompeu Fabra y Manuel de Montoliu. 8 vols. Barcelona: IEC, 19151934.

DIEC: Institut d'Estudis Catalans. Diccionari de la llengua catalana. Barcelona/Palma/València: Ed. 3 i 4/Ed. 62/Ed. Moll/Enciclopèdia Catalana/ PAM, 2000. 
DNV: Acadèmia Valenciana de la Llengua. Diccionari normatiu valencià. $<$ http://www.avl.gva.es/dnv> [enero de 2017].

Doménech Zornoza, Josep Lluís. El habla de férica. Jérica: Ayuntamiento de Jérica, 2015.

Dorca, Jordi. “Aproximació geolingüística als parlars de les Guilleries: notes de presentació". Quaderns de la Selva 22 (2010): 147-60.

Eito Mateo, Antón. El aragonés de Embún. Huesca: Publicazions d'o Consello d'a Fabla Aragonesa, 2006.

El Mole. Valencia: imp. de López, 1837, 1840-1841.

El pardal sisó, que està dalt del campanil de la església de Sent foan, atarantat al veure la barabunda que es movia en la plaza del mercat, ab motiu de les festes de la beatificació del B. Fuan de Ribera, y, lo que és més, veent els andamis que posaba el clero de Sent Joan, pensant-se anaven a fer-li una mala feta, prengué vol, y es parà en una taulada del Colegi, de a hon divisà al Dragó. València: Miguel Estevan, s. a. [1796].

El Sueco. Valencia: imp. de López, 1847.

Enguita Utrilla, José M. a "Aragón en la Edad Media: un bosquejo lingüístico". I Curso sobre lengua y literatura en Aragón (Edad Media). Ed. José M. ${ }^{2}$ Enguita. Zaragoza: Institución Fernando el Católico, 1991. 49-65.

Escobedo, Antonio. Vocabulario almeriense. Almería: Universidad de Almería, 2003.

Escrig, Josep. Diccionario valenciano-castellano. Valencia: J. Ferrer de Orga, 1851. Falla de la plassa de San Francés. Esplicasió y relasió de lo que conté esta falla. Any 1925. Valencia, 1925.

Fiestas que en el siglo IV de la canonización de san Vicente Ferrer se celebraron en Valencia. Relación escrita por Don Vicente Boix, cronista de la misma ciudad. Valencia: imp. de José Rius, 1855.

Foc y Flama 1. Valencia: imp. Tipografía Moderna, 1912.

Foz, Vicente. Prontuario del buen hablista. Zaragoza: Escar, 1903.

Frago Gracia, Juan A. "Determinación sociolingüística en la castellanización del Valle del Ebro". I Curso sobre lengua y literatura en Aragón (Edad Media). Ed. José M. ${ }^{a}$ Enguita. Zaragoza: Institución Fernando el Católico, 1991. 115-30.

Galán Castañ, Josep, y Maribel Galán Pérez. Lèxic esmortoït de la parla de Fraga. Huesca: Institut d'Estudis del Baix Cinca, 1997.

Garcés, Agliberto. Fraseología de habla popular aragonesa. Huesca: Publicaciones d'o Consello d'a Fabla Aragonesa, 2002. 
Garcés, M. ${ }^{a}$ Pilar. "La terminología del maíz en Aragón, Navarra y Rioja". Epos 5 (1989): 99-114.

García-Arista, Gregorio. Cantas baturras. Zaragoza: Manuel Sevilla, 1901.

Gargallo, José Enrique. "Una encrucijada lingüística entre Aragón, Valencia y

Castilla: el Rincón de Ademuz". Tesis doctoral. Barcelona: Universidad

Central de Barcelona, 1987.

Gargallo, José Enrique. Habla y cultura popular en el Rincón de Ademuz. Madrid: CSIC, 2004.

Gargallo Sanjoaquín, Manuel. El léxico de la ciudad de Zaragoza a mediados del siglo XX. Zaragoza: Institución Fernando el Católico, 2000.

Giralt, Javier. Lèxic de la Llitera. Lérida: Milenio, 2005.

Hernández Casajuana, Faust. L'airet de la matinà. Valencia: Manuel Pau, 1926.

Iribarren, José María. Vocabulario navarro. Pamplona: Institución Príncipe de Viana, 1952.

Jaime Gómez, José de, y José M. J Jaime Lorén. Refranero aragonés. Zaragoza: Institución Fernando el Católico, 2002.

Justes Carilla, Rosa, y Jesús Vázquez Obrador. “Aragonesismos en «La gente de mi tierra» de Crispín Botana”. Homenaje a Fosé Manuel Blecua. Huesca: Instituto de Estudios Altoaragoneses, 1986. 31-56.

La Traca: Semanari pa la chent de tro 56. Valencia: Alufre, 1886.

Lagarde, André. Le Trésor des mots d'un village occitan. Toulouse: Chez l'Auteur, 1991.

Lapesa, Rafael. Historia de la lengua española. Madrid: Gredos, 1984.

Lespy, Vastin, y Paul Raymond. Dictionnaire Béarnais ancien et moderne. 2 vols. Réimpression de l'édition de Montpellier, 1887. Genève: Slatkine Reprints, 1970.

Llatas, Vicente. El habla del Villar del Arzobispo. 2 vols. Valencia: Institución Alfonso el Magnánimo, 1959.

Llombart, Constantí. Tipos d'auca. Valencia: Vilar, 1878.

Llorente Falcó, Teodoro. Memorias de un setentón. 3 vols. Valencia: Federico Doménech, 2001.

López Allué, Luis. Obra poética (1879-1928). Huesca: La Val de Onsera, 1994. López Susín, José Ignacio, ed. El Diccionario Aragonés: colección de voces para su formación, 1902. Edición facsímil. Zaragoza: Aladrada, 2009.

Marco Yagüe, Mariano. "Vocabulario de palabras típicas". Cuadernos de Etnología de Guadalajara 41 (2004): 11-123. 
Martí, Emili, y Salvador Aparicio. El léxico de Anna. Anna: Excmo. Ayuntamiento de Anna, 1989.

Martí Gadea, Joaquim. Diccionario valenciano-castellano. Valencia: José Canales Romà, 1891.

Martí Mestre, Joaquim. Diccionari històric del valencià col-loquial. Valencia: Universitat de València, 2006.

Martí Mestre, Joaquim. "El Coloqui nou en què trata de les llàgrimes dels suaristes..., una mostra literària de les tensions entre tomistes i antitomistes en la València del darrer terç del segle XVIII". Estudis de Llengua i Literatura Catalanes 63. Barcelona: PAM, 2011. 55-83.

Martín Polo, Fernando, y Eduardo Tello Torres, eds. Historia civil, natural y eclesiástica de Titaguas de D. Simón Rojas Clemente y Rubio. Valencia: Universitat de València, 2000.

Martines, Josep. Estudi lingüístic del Diccionario valenciano de fosep Pla i Costa. Alacant/Barcelona: IIFV/PAM, 2000.

Mercadal, Manuel. Vocabulario de la Sexma de la Honor de Huesa del Común (Teruel). Zaragoza: Institución Fernando el Católico, 2004.

Miral, Domingo. Qui bien fá nunca lo pierde. Jaca: Carlos Quintilla, 1903.

Moneva y Puyol, Juan. Vocabulario de Aragón. Zaragoza: Xordica Editorial, 2004.

Monzó Nogués, Andrés. "El Mijares y el mijarense". Anales del Centro de Cultura Valenciana 19 (1951): 41-65, 93-129, 187-249.

Mora Rodenas, Ángel de. "El bienbablao": repertorio de vocablos (La Manchuela). <http://www.elbienhablao.es> [enero de 2017].

Mostolay, Chesús de. El aragonés en el Somontano de Barbastro. Zaragoza: Aneto Publicaciones, 2007.

Nagore Laín, Francho, dir. Endize de bocables de l'aragonés seguntes os repertorios lesicos de lugars y redoladas de l'Alto Aragón. 4 vols. Huesca: Instituto de Estudios Altoaragoneses, 1999.

Nagore Laín, Francho. "Bocables aragoneses «A través del Somontano Altoaragonés», de Salvador María de Ayerbe”. Homenaje a Rafael Andolz: estudios sobre la cultura popular, la tradición y la lengua en Aragón. Ed. Francho Nagore Laín. Huesca: Instituto de Estudios Altoaragoneses, 2000. 527-73.

Nagore Laín, Francho. "Notas para una caracterización lingüística del aragonés". Caplletra 32 (2002): 13-33.

Nebot Calpe, Natividad. "El castellano-aragonés en tierras valencianas (Alto Mijares, Alto Palancia, Serranía de Chelva, Enguera y la Canal de Navarrés)". Archivo de Filología Aragonesa 34-35 (1984): 395-535. 
Negredo, Marco. "O fablar de Fuenferrada (Cuencas mineras zentrals)". Luenga \& fablas 5-6 (2001-2002): 87-148.

NTLE: Lidio Nieto Jiménez y Manuel Alvar Ezquerra. Nuevo tesoro lexicográfico del español (s. XIV-1726). 11 vols. Madrid: Arco Libros, 2007.

NTLLE: Real Academia Española. Nuevo tesoro lexicográfico de la lengua española. <http://www.rae.es> [enero de 2017].

Palay, Simin. Dictionnaire du Béarnais et du Gascon modernes. París: ECNRS, 1974.

PALDC: Joan Veny. Petit Atles Lingiüstic del Domini Català. 6 vols. Barcelona: IEC, 2008-2017.

Pardo Asso, José. Nuevo diccionario etimológico aragonés. Zaragoza: imp. del Hogar Pignatelli, 1938.

Pastor Blanco, José María. Tesoro léxico de las hablas riojanas. Logroño: Universidad de La Rioja, 2004.

Pellicer, Joan. Costumari botànic. Picaña: Edicions del Bullent, 2000.

Peralta, Mariano. Ensayo de un diccionario aragonés-castellano. Zaragoza: imprenta Real, 1936.

Quintana, Artur. "El parlar de la Codonyera”. Estudis Romànics 17 (19761980): 1-253.

Rey, Alain, dir. Dictionnaire historique de la langue française. Paris: Le Robert, 2006.

Rodés, Francho, Paquita Maza y Gonzalo Gavín. Bocabulario monegrino. Samianigo: Publicazions d'o Consello d'a Fabla Aragonesa, 2005.

Romanç nou, molt graciós y entretengut, bon se referixen al peu de la lletra totes les cosetes que deuen previndre les senyoretes per a parir, la sutjecció que ban de tindre al marit y altres circunstàncies que han de guardar les casades, com vorà el curiós, en aquest any 1736. Dictat per una Musa Lapera. [s. 1.], [s. n.], [s. a.].

Romanos Hernando, Fernando. Al límite: la pervivencia del aragonés en las comarcas del norte de Zaragoza. Zaragoza: Diputación de Zaragoza, 2003.

Roselló, Roberto. "Algunes frases fetes que diem a Borriana (IV)". Elperiòdic.com. 2004. <http://www.elperiodic.com/opinion/parotets/4421> [enero de 2017].

Ruiz Marín, Diego. Vocabulario de las hablas murcianas. Murcia: Diego Marin editor, 2007.

Sacro monte parnaso de las musas católicas de los reynos de España, que unidas pretenden coronar su frente y guarnecer sus faldas con elegantes poemas en varias lenguas, en elogio del prodigio de dos mundos y flor de oriente san Francisco Xavier, de la compañia de fesús, que [...] da a la estampa el licenciado Francisco Ramón González. Valencia: Francisco Mestre, 1687. 
Sánchez Miguel, Juan Manuel. Breve diccionario del habla de los pueblos de los Montes de Toledo. Enero de 2017. <http://www.retuertadelbullaque.com/ historia/nuevo/diccionario.php>.

Sánchez Pitarch, Fernando, y Guillermo Tomás Faci. "Breve descripción del aragonés de Agüero". Archivo de Filología Aragonesa 61-62 (2005-2006): 185-216.

Serna, José S. Cómo habla La Mancha: diccionario manchego. Albacete: Grupo Altabán, 1983.

Soler Betés, Amparo-Ángeles. Replega de bocabulario de Ballobar. Huesca: Publicazions d'o Consello d'a Fabla Aragonesa, 2004.

Torres Fornes, Cayetano. Sobre voces aragonesas usadas en Segorbe. València: Miguel Gimeno, 1903.

Un pillo y els chics educats en la casa de Benefisènsia, y lo que serien moltísims chics a no haber este benèfic estaliment. Tres coloquis. Valencia: Laborda, 1846.

Veny, Joan. Mots d'abir y mots d'avui. Barcelona: Empúries, 1991.

Vidal i Roig, Francesc. La ovella descarrià. Valencia: imp. de T. Soto, 1903. 